\title{
Gut besuchter Auftakt
}

\section{Der 1. Pflegetag Rheinland-Pfalz}

" Mit rund 1.200 Besuchern und über 50 Ausstellern fand am 3.5.2017 der erste Pflegetag Rheinland-Pfalz in Mainz statt. Die rheinland-pfälzische Ministerpräsidentin Malu Dreyer hob in ihrem Grußwort die gesamtgesellschaftliche Bedeutung beruflich Pflegender hervor und bekräftigte die Notwendigkeit einer institutionellen Interessenvertretung in Form einer Pflegekammer. Sie betonte, dass sich die Landesregierung mit ihrer Pflegepolitik für die Einführung einer generalistischen Pflegeausbildung einsetze, die den Bedarfen der Zukunft gerecht werde.

Die Landespflegekammer Rheinland-Pfalz als Veranstalter legte den Fokus des Pflegetags auf pflegefachliche Themen und regionale Projekte. Auf einer „Kammermeile“ informierte sie die Besucher über ihre Arbeit. Gleichwohl nutzten Kammerpräsident Dr. Markus Mai und Kammervizepräsidentin Sandra Postel im Vorfeld des Pflegetags die Gelegenheit, ihre Forderungen an Politik und Arbeitgeber zu formulieren. Auf der Agenda stehen dabei drei zentrale Themen, bei denen es aus ihrer Sicht nach wie vor dringenden Handlungsbedarf gibt:

\section{Krankenhausinvestitionen der Landesregierung}

Die Fördermittel des Landes für die Kliniken seien seit 2002 von 122 Mio. $€$ auf 114 Mio. € pro Jahr gesunken, während der Baupreisindex im gleichen Zeitraum um 35\% stieg. Preisbereinigt hätten damit im Jahr 2016 nur noch 60\% der Investitionsmittel des Jahres 2002 zur Verfügung gestanden. Mittel in einer Höhe, die schon damals nicht für eine vollständige Ausfinanzierung gereicht hätten. Die liege an der im Vergleich zu anderen Branchen dramatisch niedrigen Investitionsquote von $3,2 \%$. Mai konstatierte, dass das Land seiner Verpflichtung in diesem Punkt noch nicht ausreichend nachkomme. Er befürchtet eine weitere Verschlechterung der Finanzlage der Krankenhäuser in Rheinland-Pfalz und bemängelt, dass darunter nach wie vor auch die Pflegepersonalbesetzung zu leiden habe. Auch wenn der Haushalt gerade verabschiedet worden sei - für die Pflegekammer bleibe die Investitionsfinanzierung weiterhin ein vordringliches Thema bei ihren Gesprächen mit der Politik. Die von der „Ampel-Koalition" verabredete Aufstockung bis 2020 um dann mind. 15 Mio. Euro, könne aufgrund des lang anhaltenden Investitionsstaus nur ein Tropfen auf den heißen Stein sein. Mai fordert einen Investitionspakt auf Bundesebene mit der Deutschen Krankenhausgesellschaft (DKG).

\section{Personalbemessung}

Für eine zwingend gesetzlich verankerte, längst überfällige Regelung zur Personalbemessung mit den Faktoren Personalstärke und Qualifikation macht sich Sandra Postel stark. Grundlage müssten pflegerische Qualitätsnormen sein. Postel sieht eine gesicherte, bedarfsgerechte Patientenversorgung bereits jetzt gefährdet. Pflegende stünden bei der derzeitigen Arbeitssituation in allen Sektoren enorm unter Druck. „Pflege kompensiert", so ihre Einschätzung. Die Pflegekammer fordert eine zügige Verbesserung der Rahmenbedingungen, die umgehende Senkung der Arbeitsbelas-

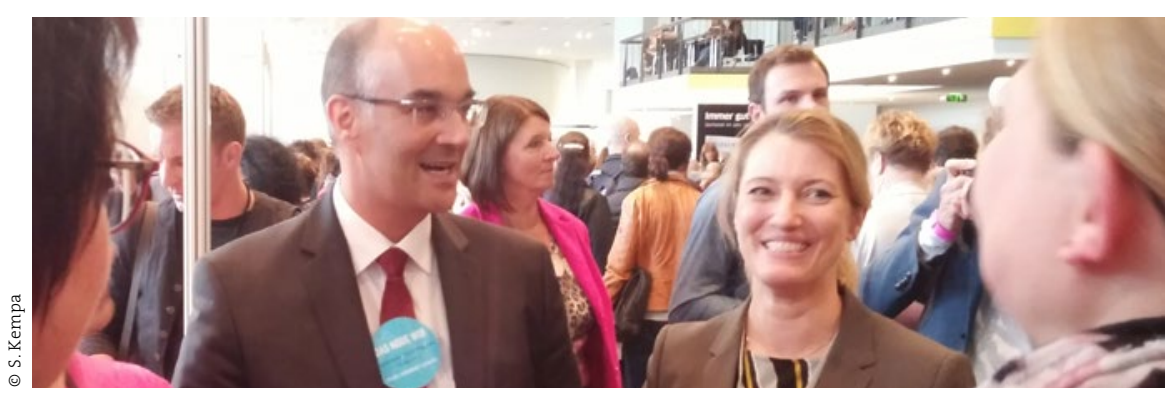

tung für Pflegende und verlangt von der Politik, mehr Verantwortung für die pflegerische Versorgung der Gesellschaft der Zukunft zu übernehmen. „Wir wollen uns wieder um die Pflegebedürftigen kümmern können und die notwendige Zeit dafür haben", so Postels Appell. Dabei sei die beschlossene Einführung von Personaluntergrenzen im Krankenhaus ein erster kleiner Schritt. „Die Definition von Mindestpersonalausstattungen hilft aber nicht, wenn es darum geht, eine den Pflegebedarfen angemessene Personalvorhaltung sicherzustellen", so Postel kritisch. Bei den unterschiedlichen Interessen der Selbstverwaltungspartner sei es fraglich, ob sie sinnvolle und für die Versorgung hochwertige Lösungen auf den Weg bringen werden.

\section{Arbeitsbedingungen / tarifliche Verbesserungen in der Altenpflege}

Seit 2015 bekommen Arbeitgeber, die ihre Beschäftigten nach Tarifvertrag bezahlen, die Gehälter von den Kostenträgern in der Pflegevergütung refinanziert. Die Pflegestärkungsgesetze bieten Einrichtungsträgern weitere Möglichkeiten, die Rahmenbedingungen für Pflegende zu verbessern. Das PSG III sieht seit Anfang 2017 eine Anlehnung an tarifliche Bestimmungen vor, die bislang für nicht-tarifgebundene so nicht vorhanden gewesen sei. In einem Brief an ca. 300 private Einrichtungen der Altenpflege forderte die Pflegekammer Rheinland-Pfalz daher im April 2017 die Arbeitgeberseite auf, die Rahmenbedingungen für die dort beschäftigen Pflegenden adäquat zu gestalten und $u$. a. tarifliche Bezahlung zu ermöglichen. Einkommensunterschiede, die allein aus der Beschäftigung in unterschiedlichen Einrichtungen resultierten, seien nicht länger hinnehmbar. Den Gewerkschaften bietet die Landespflegekammer ausdrücklich die Zusammenarbeit an, um in diesem wichtigen Thema „an einem Strang zu ziehen“. (sk)

Pressekonferenz „1. Pflegetag

Rheinland-Pfalz"; Mainz, 2. Mai 2017

(www.pflegekammer-rlp.de) 